\title{
Immaturity and Disease Severity are Independent Risk Factors to Develop Retinopathy of Prematurity
}

\author{
K. Allegaert ${ }^{*}$, , S. Vanhaesebrouck ${ }^{1}$, C. Vanhole ${ }^{1}$ and I. Casteels ${ }^{2}$ \\ ${ }^{I}$ Neonatal Intensive Care Unit, Division Women and Child, University Hospital, Herestraat 49, 3000 Leuven, Belgium \\ ${ }^{2}$ Department of Ophthalmology, University Hospital, Leuven, Belgium
}

\begin{abstract}
Purpose: Document indicators of an increased risk to develop retinopathy of prematurity (ROP) in low birth weight (LBW, $<1500 \mathrm{~g}$ ) infants.

Methods: Retrospective chart review. Neonatal characteristics collected were birth weight, gestational age (GA), CRIB (Clinical Risk Index for Babies) score, Apgar score and indicators of respiratory, circulatory and renal instability. Small for gestational age (SGA, i.e. $<10^{\text {th }}$ percentile GA) was registered while postnatal growth was also recorded as a dichotomous variable, whether or not $150 \%$ of the birth weight was reached on day 42 of life.

Results: On 284 LBW infants, 248 (87\%) survived until discharge. ROP (any stage) was documented in 74 (30\%) survivors of whom 41 (17\%) developed stage 3 ROP. Incidence of stage 3 ROP in $<1000 \mathrm{~g}$ and ROP in 1000-1250 birth weight cohorts was $42 \%$ and $16 \%$ respectively with almost absence of any ROP in neonates with a birth weight $>1250 \mathrm{~g}$. In a logistic regression model in 248 LBW survivors, both GA (OR 0.42) and CRIB score (OR 1.35) remained independent risk factors for stage $3 \mathrm{ROP}$, resulting in $90 \%$ of cases classified correctly.

Conclusions: Stage 3 ROP is almost exclusively limited to neonates with a birth weight below 1000g and retinopathy (any stage) is almost absent in neonates with a birth weight $>1250 \mathrm{~g}$. Immaturity, i.e. GA at birth and CRIB score, reflecting disease severity, are the most important risk factors to develop retinopathy.
\end{abstract}

\section{INTRODUCTION}

Retinopathy of prematurity (ROP) is a multifactorial disease with numerous risk factors either based on immaturity (gestational age, birth weight) or indicators of disease severity [1]. Such indicators besides birth weight or gestational age (GA) might be used to further discriminate the relative risk to develop ROP associated with a given GA or birth weight $[2,3]$. Prenatal as well as postnatal growth restriction, CRIB score, renal failure, dopamine administration and indicators of respiratory disease were reported in literature to be associated with an increased risk to develop ROP [4-14]. Observations on the simultaneous analysis of all these risk factors to unveil the most prominent risk factors are limited.

O'Connor et al. recently reviewed the ophthalmological problems associated with preterm birth and hereby reemphasized the long term consequences of retinopathy following preterm birth on visual function, eye growth and visual impairment in later life [15]. Others reported on the potential relevance of systematic screening and earlier surgical intervention to prevent further deterioration of retinopathy and visual function [16, 17]. Therefore, additional indicators might also enable caregivers to decrease the number of screening procedures in neonates at very low

*Address correspondence to this author at the Neonatal Intensive Care Unit, Division of Women and Child, University Hospital Gasthuisberg, Herestraat 49, 3000 Leuven, Belgium; Tel: 00-32-16-343210; Fax: 00-32-16-343209; E-mail: karel.allegaert@uz.kuleuven.ac.be risk or to develop secondary preventive strategies in neonates at high risk to develop major ROP [16]. Finally, these additional risk factors might help us to understand the underlying pathogenesis $[1-3,15,18,19]$. To disentangle the various risk factors associated with ROP during later neonatal life, associations between perinatal variables and ROP were investigated in a cohort of low birth weight (LBW, < 1500g) neonates admitted in one single neonatal intensive care unit (NICU).

\section{MATERIALS AND METHODOLOGY}

\section{Population}

A retrospective chart review in low birth weight infants (i.e. < 1500g) admitted in the neonatal intensive care unit, Gasthuisberg, during a 3 year's period was performed. Infants had to be admitted in the unit within 6 hours after birth, needed to stay in the unit during at least the first week of life and ophthalmologic screening needed to be performed until full retinal vascularisation was documented to be included in this study. Neonatal characteristics collected at birth in these infants were birth weight, GA and Apgar score at five minutes. GA was calculated using the expected date of delivery based on an ultrasound performed before 20 weeks' gestation, or if not available, was based on the last mother's period and on neonatal clinical findings. Birth weight was documented on admission and compared with the birth weight of a Flemish cohort of preterm neonates as published by the Study Center of Perinatal Epidemiology to document SGA $\left(<10^{\text {th }}\right.$ percentile for a given GA) [20]. Appropriated postnatal growth was registered as a dichotomous variable, defined as either or not an additional 
$50 \%$ increase compared to the initial birth weight on day 42 of postnatal life was reached [4, 6]. The CRIB (Clinical Risk Index for Babies, i.e. a disease severity scoring system) score was originally developed to express the relative mortality risk but there are reports on the association of CRIB and long term morbidity [7, 8]. Respiratory characteristics collected were either markers of duration of respiratory disease (last day of ventilation, last day of respiratory support, last day of supplemental oxygen) or were markers of severity of respiratory disease in the first 72 hours of life: intubation (yes/no), maximal Mean Airway Pressure (MAP) and Oxygenation Index (OI) at maximal MAP [MAP x $100 \mathrm{x}$ fractional oxygen/arterial oxygen $(\mathrm{mmHg})]$. Prescriptive characteristics of dopamine (hours of administration in the first week of life) was used as marker of cardiovascular instability and maximal creatinaemia $(\mathrm{mg} / \mathrm{dl})$ in the first week of life was registered as indicator of renal dysfunction/failure [8, 13, 14].

\section{Ophthalmologic Screening}

Ophthalmologic screening was performed by indirect fundoscopy. First ophthalmologic screening was performed at the postnatal age of 4 to 5 weeks and findings were classified according to the International Classification of Retinopathy of Prematurity. In case of a normal fundoscopy, a two weeks interval examination was performed until full vascularisation. If ROP stage 1 or 2 was diagnosed, the baby was examined on a weekly basis. In case of ROP stage 3, fundoscopy was performed twice a week. If infants fulfilled Cryo-ROP criteria, i.e. threshold disease (at least five contiguous or eight cumulative clock hours of stage 3 ROP in zone 1 or 2 in the presence of Plus disease) retinal surgery was performed [21].

\section{Statistics}

Results were reported by mean and standard deviation, median and range or incidence. Since ROP is a marker of neonatal morbidity during later neonatal stay, only clinical characteristics in survivors were used to evaluate potential risk factors (monovariate). A logistic regression model was subsequently applied to document the independent contributors to stage 3 ROP in LBW infants. A similar approach (monovariate analysis with subsequent logistic regression) was also performed in two subgroups of neonates. Firstly, ELBW, i.e. $<1000 \mathrm{~g}$ infants who developed stage 3 were compared to ELBW infants who did not develop stage 3 ROP. Secondly, in neonates with a birth weight between 1000 and $1250 \mathrm{~g}$, observations in neonates who either or not developed any ROP were analysed.

A p-value below 0.05 was considered significant.

\section{RESULTS}

On 284 LBW neonates admitted with a birth weight below $1500 \mathrm{~g}, 248(87 \%)$ survived until discharge. Nonsurvivors $(\mathrm{n}=36)$ had a significant lower birth weight $(744$, range $320-1320 \mathrm{~g}$ compared to 1127 , range $400-1500 \mathrm{~g}$ ) and GA (25.5, range 23-36 compared to 29 , range $24-36$ weeks) while the CRIB Score (10, range 2-22 compared to 2, range $0-15$ ) and the maximal OI observed in the first 72 hours of life was significantly higher (19.9, range 2.3 to 95.4 compared to 7.2 , range 0 to 32.9) (all at least $\mathrm{p}<0.01$ ) in non-survivors. A complete set of clinical data, including ROP follow up data were available in 245 LBW survivors. ROP (any stage) was documented in 74/248 (30\%) survivors of which $41(17 \%)$ developed stage 3 ROP. GA-based incidence of any ROP or stage 3 ROP are presented in Fig. (1). Characteristics of LBW survivors who developed stage 3

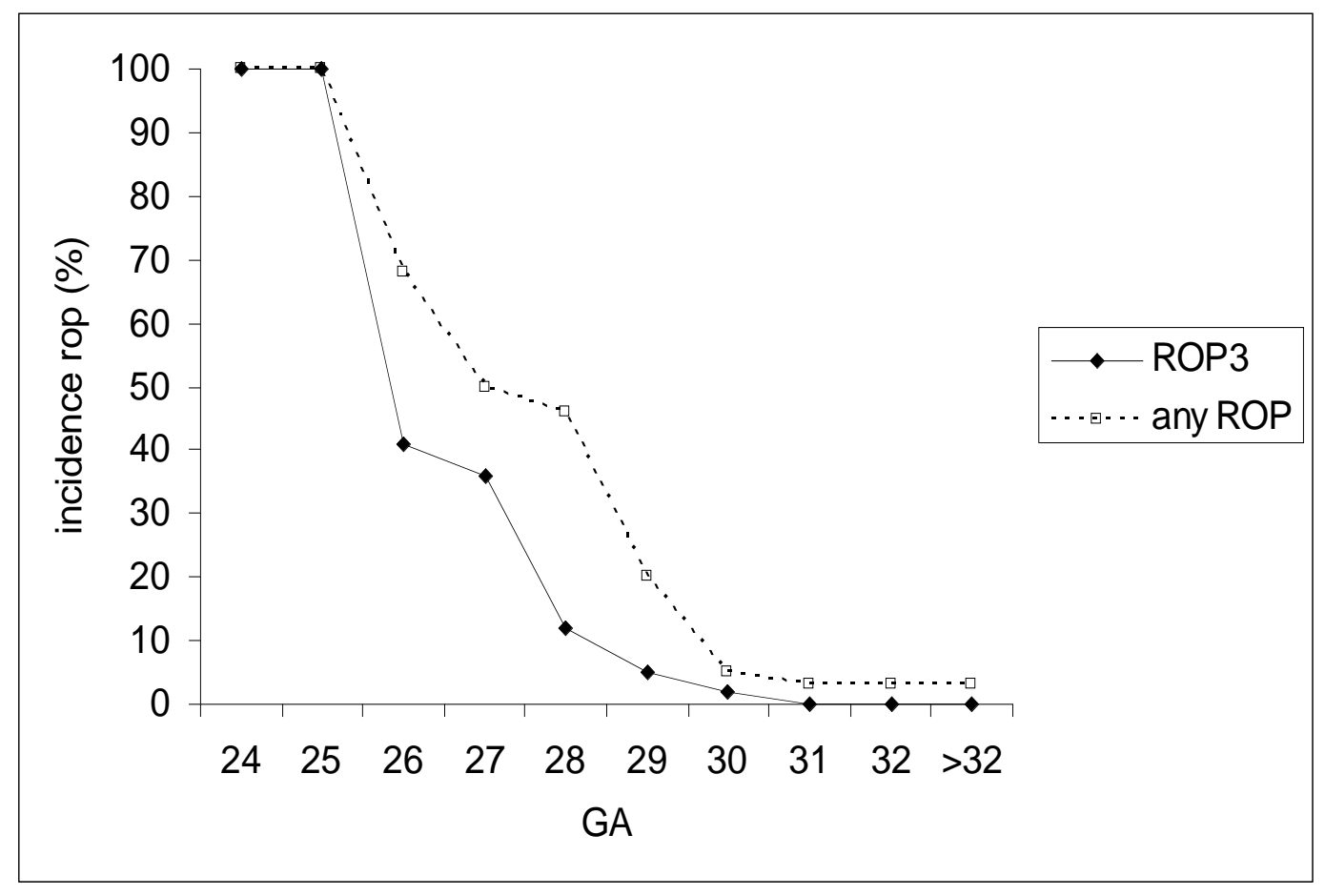

Fig. (1). Gestational age based relative risk (\%) to develop stage 3 ROP (continuous line, black boxes) and any retinopathy (dotted line, white boxes) in this single unit cohort $(2000-2002)$ of survivors $(n=248)$ with a birth weight below $1500 \mathrm{~g}$. (X-axis: gestational age). 
Table 1. Clinical Characteristics of LBW Neonates (i.e. $<1500$ ) who Developed Stage 3 ROP are Compared to those who did not Develop Stage 3 ROP

\begin{tabular}{|c|c|c|c|}
\hline & ROP, Stage 3 & No Stage 3 ROP & p-Value \\
\hline Number & 41 & 204 & \\
\hline Gestational age (weeks) & $26(24-30)$ & $30(25-6)$ & $<0.01$ \\
\hline Weight at birth & $715(400-1280)$ & $1200(480-1500)$ & $<0.01$ \\
\hline Growth restriction at birth $\left(<10^{\text {th }}\right)$ & $43 \%$ & $27 \%$ & NS \\
\hline $150 \%$ of birth weight on day 42 & $20 \%$ & $14 \%$ & $<0.05$ \\
\hline Apgar score 5 minutes & $8(2-10)$ & $8(4-10)$ & NS \\
\hline CRIB score & $8(1-13)$ & $2(0-13)$ & $<0.01$ \\
\hline Blood culture proven infection & $87 \%$ & $44 \%$ & $<0.01$ \\
\hline Ventilated during neonatal stay & $98 \%$ & $63 \%$ & $<0.01$ \\
\hline Surfactant administration & $93 \%$ & $49 \%$ & $<0.01$ \\
\hline Duration of respiratory support (days) & $15(0-71)$ & $3(0-42)$ & $<0.01$ \\
\hline Duration of oxygen administration (days) & $66(8-139)$ & $11(0-144)$ & $<0.01$ \\
\hline Maximal oxygenation index $0-72$ hours & $4.9(1.6-20.5)$ & $3.8(0.7-15.2)$ & $<0.05$ \\
\hline Duration of dopamine administration first week & $52(0-168)$ & $0(0-168)$ & $<0.01$ \\
\hline Creatinaemia (mg/dl) & $1.19(0.5-2.19)$ & $1.06(0.5-2.22)$ & $<0.01$ \\
\hline
\end{tabular}

ROP were compared to survivors without stage 3 ROP (Table 1). In a logistic regression model with stage 3 ROP as dependent variable, $\mathrm{GA}(\mathrm{OR}=0.42,95 \%$ C.I. 0.28 to 0.62$)$ and CRIB score (OR 1.35, 95\% CI 1.14 to 1.6) remained independent contributors, resulting in $90 \%$ of cases classified correctly.

When only observations in ELBW (i.e. < 1000g) neonates were considered $(n=95)$, incidence of ROP and stage 3 ROP was $65 \%$ and $42 \%$ respectively. Characteristics of ELBW (i.e. $<1000 \mathrm{~g}$ ) neonates who developed stage 3 ROP $(n=40)$ were compared to those who did not develop stage 3 ROP $(n=55)$. GA and birth weight, CRIB score and indicators of disease severity/duration were significantly different, but neither pre- nor postnatal growth restriction further contributed in this overall high risk population. In a logistic regression model with stage 3 ROP as dependent variable, only GA $(\mathrm{OR}=0.44,95 \%$ C.I. 0.25 to 0.64$)$ and birth weight (O.R. 0.98, 95\% CI 0.97-0.99) remained independent contributors, resulting in $77 \%$ of cases classified correctly.

Finally, characteristics of neonates $(n=54)$ with a birth weight between $1000 \mathrm{~g}$ and $1250 \mathrm{~g}$ who developed any stage of ROP $(n=9)$ were compared to those who did not develop ROP $(n=45)$. In addition to various indicators of disease severity/duration, postnatal growth restriction was also associated with an increased risk to develop retinopathy (any stage) (Table 2). In a logistic regression model with any ROP as dependent variable, only duration of oxygen administration remained an independent risk factor (OR $1.05,95 \%$ C.I. 1.02 to 1.07 ) to develop any ROP, resulting in $85 \%$ of cases classified correctly.

\section{DISCUSSION}

Based on the analysis of this single unit cohort, it was documented that the GA-based relative risk to develop ROP in LBW-infants has a logarithmic or bimodal distribution in line with other European population studies. This distribution probably can be explained by the major impact of immaturity in ELBW neonates while in relatively more mature infants (1000-1250g), ROP likely is the final result of a combined effect of preterm birth and disease severity, reflected by the impact of both GA and CRIB score on the risk to develop stage 3 ROP in this cohort of LBW infants $[1,2,7,8]$.

When the complete cohort of observations in LBW infants were analysed, GA and CRIB score both were independent indicators of the risk to develop retinopathy. Immaturity or birth at the threshold of viability has repeatedly been reported to be the most significant risk factor to develop stage 3 ROP [2, 3, 5, 10]. Even in the ELBW group ( $<1000 \mathrm{~g}$ birth weight), GA remained a risk factor to develop stage $3 \mathrm{ROP}$ in line with the earlier reported EpiBel study cohort [10]. The CRIB score was also associated with an increased risk to develop stage 3 retinopathy. CRIB score was originally designed to quantify the relative risk of neonatal mortality but others linked CRIB score with morbidity characteristics, including ROP, during later neonatal stay [7].

In the logistic regression model in the 'low risk' subgroup (1000-1250g), only duration of supplementary oxygen administration was an independent indicator of an increased risk to develop any stage of ROP, resulting in still $85 \%$ of cases classified correctly. The association of respiratory disease severity and duration with an increased 
Table 2. Clinical Characteristics of ELBW Neonates (i.e. $<1000$ g) who Developed Grade 3 ROP are Compared to those who did not Develop Grade 3 ROP

\begin{tabular}{|c|c|c|c|}
\hline & ROP, Grade 3 & No Grade 3 ROP & p-Value \\
\hline Number & 40 & 55 & \\
\hline Gestational age (weeks) & $26(24-29)$ & $28(25-33)$ & $<0.01$ \\
\hline Weight at birth & $690(400-970)$ & $825(480-1000)$ & $<0.01$ \\
\hline Growth restriction at birth $\left(<10^{\text {th }}\right)$ & $45 \%$ & $45 \%$ & NS \\
\hline $150 \%$ of birth weight on day 42 & $18 \%$ & $15 \%$ & NS \\
\hline Apgar score 5 minutes & $8(2-10)$ & $8(4-10)$ & NS \\
\hline CRIB score & $8(1-13)$ & $4(1-13)$ & $<0.01$ \\
\hline Blood culture proven infection & $88 \%$ & $64 \%$ & NS \\
\hline Ventilated during neonatal stay & $97 \%$ & $84 \%$ & NS \\
\hline Surfactant administration & $95 \%$ & $67 \%$ & $<0.05$ \\
\hline Duration of respiratory support (days) & $15.5(0-71)$ & $6(0-42)$ & $<0.05$ \\
\hline Duration of oxygen administration (days) & 66 (SD 27) & 43 (SD 25) & $<0.01$ \\
\hline Maximal oxygenation index $0-72$ hours & $8.8($ SD 5.9) & $11.1(\mathrm{SD} 7.1)$ & $<0.05$ \\
\hline Duration of dopamine administration first week & $48(0-168)$ & $6(0-168)$ & $<0.01$ \\
\hline Creatinaemia (mg/dl) & $1.19(0.5-1.7)$ & $1.15(0.66-2.2)$ & NS \\
\hline
\end{tabular}

risk to develop ROP reconfirms earlier reports and in a strong argument in favour of further research in the common pathogenetic mechanisms involved [1, 2, 19, 21, 22]. In the low risk $(\mathrm{n}=54,1000 \mathrm{~g}-1250 \mathrm{~g}$ ) subgroup (Table 3), 19 neonates displayed postnatal growth restriction, of whom 9 also developed ROP. Therefore, postnatal growth restriction had limited sensitivity (40\%) but moderate specificity $(87 \%)$ to predict ROP. There are observations on the impact of postnatal growth restriction in animal experimental setting and the associated risk to develop ROP while in human preterm neonates, postnatal insulin-like growth factor 1 (IGF-1) deficiency is associated with both restricted postnatal growth and retinopathy of prematurity $[3-6,9,18$, 19].

The current observations at least suggest that the 'normalisation' of postnatal growth (i.e. $150 \%$ of birth

Table 3. Clinical Characteristics of Neonates $(1000$ - 1250 g) who Developed any Grade of ROP are Compared to those who did not Develop ROP

\begin{tabular}{|c|c|c|c|}
\hline & Retinopathy & No Retinopathy & p-Value \\
\hline Number & 9 & 45 & \\
\hline Gestational age (weeks) & $30(26-33)$ & $28(26-32)$ & NS \\
\hline Weight at birth & $1130(1025-1250)$ & $1080(1030-1250)$ & NS \\
\hline Growth restriction at birth $\left(<10^{\text {th }}\right)$ & $11 \%$ & $28 \%$ & NS \\
\hline $150 \%$ of birth weight on day 42 & $44 \%$ & $14 \%$ & $<0.05$ \\
\hline Apgar score 5 minutes & $8(5-10)$ & $8(7-9)$ & NS \\
\hline CRIB score & $2(0-7)$ & $2(1-6)$ & NS \\
\hline Blood culture proven infection & $44 \%$ & $42 \%$ & NS \\
\hline Surfactant administration & $77 \%$ & $37 \%$ & $<0.01$ \\
\hline Ventilated during neonatal stay & $100 \%$ & $60 \%$ & NS \\
\hline Duration of respiratory support (days) & $19(0-54)$ & $8(0-46)$ & $<0.01$ \\
\hline Duration of oxygen administration (days) & $49(6-105)$ & $7.5(0-70)$ & $<0.01$ \\
\hline Maximal oxygenation index $0-72$ hours & $12.8(5.8-16.3)$ & $10.8(5.6-16.3)$ & $<0.05$ \\
\hline Duration of dopamine administration first week & $28(0-144)$ & $0(0-112)$ & $<0.05$ \\
\hline Creatinaemia (mg/dl) & $1.08(0.54-1.71)$ & $1.08(0.89-1.82)$ & NS \\
\hline
\end{tabular}


weight on day 42) has no independent impact on the risk to develop ROP. However, the absence of any independent effect of postnatal growth restriction on retinopathy should be interpreted cautiously, since postnatal weight gain is only a rough indicator of nutritional balance. It is not unlikely that qualitative aspects like vitamin E, iron load and intake, glycaemia or the use of human milk are more of relevance in ELBW infants who still display an overall high risk to develop ROP in the present cohort [3, 9, 11, 12]. Finally, the need for dopamine administration or renal dysfunction reconfirmed earlier findings that both might serve as indicators of increased risk to develop ROP, but neither dopamine administration, nor renal dysfunction (creatinaemia) were independent risk factors to develop ROP $[8,13,14]$.

\section{CONCLUSION}

Based on observations collected in a cohort of $245 \mathrm{LBW}$ survivors (i.e. $<1500 \mathrm{~g}$ ), stage 3 ROP was almost exclusively limited to neonates with a birth weight below $1000 \mathrm{~g}$ and retinopathy (any stage) was almost absent in neonates with a birth weight $>1250 \mathrm{~g}$. Immaturity, i.e. GA at birth and CRIB score, reflecting disease severity, are the most important risk factors to develop retinopathy in LBW infants.

\section{ACKNOWLEDGEMENTS}

The clinical research of $\mathrm{K}$ Allegaert is supported by the Fund for Scientific Research, Flanders (Belgium) (FWO Vlaanderen) by a Fundamental Clinical Investigatorship $(1800209 \mathrm{~N})$.

\section{REFERENCES}

[1] Sylverster CL. Retinopathy of prematurity. Semin Ophthalmol 2008; 23: 318-23.

[2] Larsson E, Carle-Petrelius B, Cernerud G, et al. Incidence of ROP in two consecutive Swedish population based studies. $\mathrm{Br} \mathrm{J}$ Ophthalmol 2002; 86: 1122-6.

[3] Chen J, Smith LE. Retinopathy of prematurity. Angiogenesis 2007; 10: 133-40.

[4] Wallace DK, Kylstra JA, Phillips SJ, Hall JG. Poor postnatal weight gain: a risk factor for severe retinopathy of prematurity. J AAPOS 2000; 4: 343-7.

[5] Filho JB, Bonomo PP, Maia M, Procianoy RS. Weight gain measured at 6 weeks after birth as a predictor for severe retinopathy of prematurity: study with 317 very low birth weight preterm babies. Graefes Arch Clin Exp Ophtalmol 2008; (online Dec 4 2008).
[6] Allegaert K, Vanhole C, Casteels I, et al. Perinatal growth characteristics and associated risk to develop threshold retinopathy of prematurity. J AAPOS 2003; 7: 34-7.

[7] Yang MB, Donovan EF, Wagge JR. Race, gender and clinical risk index for babies (CRIB) score as predictors of severe retinopathy of prematurity. J AAPOS 2006; 10: 253-61.

[8] Allegaert K, Casteels I, Cossey V, Devlieger H. Retinopathy of prematurity: any difference in risk factors between a high and a low risk population? Eur J Ophthalmol 2003; 13: 784-8.

[9] Hikino S, Ihara K, Yamamoto J, et al. Physical growth and retinopathy in preterm infants: involvement of IGF-1 and GH. Pediatr Res 2001; 50: 732-6.

[10] Allegaert K, de Coen K, Devlieger H. The EpiBel study group. Threshold retinopathy at threshold of viability: the Epibel study. $\mathrm{Br}$ J Ophthalmol 2004; 88: 239-42.

[11] Garg R, Agthe AG, Donohue PK, Lehmann CU. Hyperglycemia and retinopathy of prematurity in very low birth weight infants. J Perinatol 2003; 23: 186-94.

[12] Hylander MA, Strobino DM, Pezzullo JC, Dhanireddy R. Association of human milk feedings with a reduction in retinopathy of prematurity among very low birth weight infants. J Perinatol 2001; 21: 356-62.

[13] Mizoguchi MB, Chu TG, Murphy FM, et al. Dopamine use is an indicator for the development of threshold retinopathy of prematurity. Br J Ophthalmol 1999; 83: 425-8.

[14] Allegaert K, Cossey V, Naulaers G, et al. Dopamine is an indicator but not an independent risk factor for stage 3 retinopathy of prematurity in extreme low birthweight infants. Br J Ophthalmol 2004; 88: 309-10.

[15] O'Connor AR, Wilson CM, Fielder AR. Ophthalmological problems associated with preterm birth. Eye 2007; 21: 1254-60.

[16] Section on Ophthalmology American Academy of Pediatrics; American Academy of Ophthalmology; American Association for Pediatric Ophthalmology and Strabismus. Screening examination of premature infants for retinopathy of prematurity. Pediatrics 2006; 117: 572-6.

[17] Early treatment for Retinopathy of Prematurity Cooperative Group. Revised indications for the treatment of retinopathy of prematurity: results of the early treatment for retinopathy of prematurity randomized trial. Arch Ophthalmol 2003: 121; 1684-94.

[18] Smith LE. IGF-1 and retinopathy of prematurity in the preterm infant. Biol Neonate 2005; 88: 237-44.

[19] Vanhaesebrouck S, Daniels H, Moons L, et al. Oxygen-induced retinopathy in mice: amplification by neonatal IGF-I deficit, and attenuation by IGF-I administration. Pediatr Res 2008; (online, Dec 10 2008).

[20] Devlieger H, Martens G, Bekaert A, Eeckels R. Standards of birth weight for gestational age in Flanders. Tijdsch Geneesk 2000; 56: $1-14$.

[21] Committee for the classification of Retinopathy of Prematurity. An international classification of retinopathy of prematurity. Arch Ophthalmol 1984; 102: 1130-4.

[22] Saugstad OD. Oxidative stress in the newborn - a 30-year perspective. Biol Neonate 2005; 88: 228-36. 